\title{
The effect of ageostrophy on the stability of thin oceanic vortices
}

\author{
E.S. Benilov \\ Department of Mathematics, University of Limerick, Room B2025, Limerick, Ireland
}

Received 17 August 2004; received in revised form 20 December 2004; accepted 14 January 2005

Available online 26 March 2005

\begin{abstract}
This paper examines the stability of vortices in a two-layer ocean on the $f$-plane. The mean depth $\bar{h}_{1}$ of the upper layer is assumed to be much smaller than the depth $\bar{h}_{2}$ of the lower layer. Using the primitive equations, we derive an asymptotic criterion for baroclinic instability of compensated (i.e. confined to the upper layer) vortices. Surprisingly, it coincides exactly with a similar criterion derived from the quasigeostrophic equations [Benilov, E.S., 2003. Instability of quasigeostrophic vortices in a two-layer ocean with thin upper layer. J. Fluid Mech. 475, 303-331]. Thus, to leading order in $\bar{h}_{1} / \bar{h}_{2}$, ageostrophy does not affect the stability properties of thin compensated vortices. As a result, whether a vortex is stable or not, depends on its shape, not amplitude (although the growth rate of an unstable vortex does depend on its amplitude).
\end{abstract}

(c) 2005 Elsevier B.V. All rights reserved.

Keywords: Ageostrophy; Ocean vortices ; Baroclinic instability

\section{Introduction}

It is well-known that oceanic vortices (rings) exist for years, whereas almost all theoretical work indicates that they are unstable. This contradiction has been identified more than 20 years ago, but only recent advances appear to bring it close to resolution. First, Dewar and Killworth (1995) have demonstrated that the stability properties of rings are very sensitive

E-mail address: eugene.benilov@ul.ie (E.S. Benilov). 
to the 'deep flow' (weak circulation in the lower layer). Then, Benilov (2004) found a particular type of deep flow that always stabilizes the vortex, and also argued that it arises naturally below oceanic rings.

Still, before one could be convinced that the deep flow is responsible for stability of rings, it should be demonstrated that the simplest model - that of compensated (i.e. confined to the upper layer) vortices-is exhausted beyond reasonable doubt. And this has not been done yet, as it is still unclear whether or not a compensated vortex can be stabilized by ageostrophic effects.

The present paper examines the stability of an ageostrophic vortex in a two-layer ocean with thin upper layer (most 'real' rings are indeed localized in a relatively thin layer). A very similar problem, but for a quasigeostrophic (QG) vortex, has been previously studied by Benilov (2003) - and comparison with his results will allow us to find out exactly what influence ageostrophy has on the stability of rings.

In the next section, we shall formulate the linearized equations for harmonic disturbances (eigenmodes), superposed on a compensated vortex in a two-layer ocean. In Section 3, we shall classify the eigenmodes, and, in Sections 4 and 5, examine their stability.

\section{Governing equations}

Consider a two-layer ocean on the $f$-plane with rigid lid and flat bottom, and let the layers' densities be $\rho_{1,2}$ (where 1 marks the upper layer). We shall use polar coordinates $(r, \theta)$ and characterize the motion of the layers by the radial velocities $u_{1,2}$, swirl velocities $v_{1,2}$, pressures $p_{1,2}$ and depths $h_{1,2}$ of the layers. Finally, we shall introduce the time variable $t$.

Assume that the layers have non-zero mean depths $\bar{h}_{j}(j=1,2$ is the layer number $)$ and define the deformation radius based on $\bar{h}_{1}$,

$$
R_{\mathrm{d}}=\frac{\sqrt{g^{\prime} \bar{h}_{1}}}{f_{0}}
$$

where $g^{\prime}=g\left(\rho_{2}-\rho_{1}\right) / \rho_{2}$ is the reduced gravity and $f_{0}$ is the Coriolis parameter. Then, we can introduce the following non-dimensional variables:

$$
\begin{aligned}
& t_{*}=f_{0} t, \quad r_{*}=\frac{r}{R_{\mathrm{d}}}, \quad \theta_{*}=\theta, \quad\left(u_{j *}, v_{j *}\right)=\frac{\left(u_{j}, v_{j}\right)}{\sqrt{g^{\prime} \bar{h}_{1}}}, \\
& p_{j *}=\frac{p_{j}}{\rho_{2} g^{\prime} \bar{h}_{1}}, \quad h_{j *}=\frac{h_{j}}{\bar{h}_{j}} .
\end{aligned}
$$

Omitting the asterisks, we can write the governing equations in the form

$$
\begin{aligned}
& \frac{\partial u_{j}}{\partial t}+u_{j} \frac{\partial u_{j}}{\partial r}+\frac{1}{r} v_{j}\left(\frac{\partial u_{j}}{\partial \theta}-v_{j}\right)+\frac{\partial p_{j}}{\partial r}=v_{j}, \\
& \frac{\partial v_{j}}{\partial t}+u_{j} \frac{\partial v_{j}}{\partial r}+\frac{1}{r} v_{j}\left(\frac{\partial v_{j}}{\partial \theta}+u_{j}\right)+\frac{1}{r} \frac{\partial p_{j}}{\partial \theta}=-u_{j},
\end{aligned}
$$




$$
\begin{aligned}
& r \frac{\partial h_{j}}{\partial t}+\frac{\partial}{\partial r}\left(r u_{j} h_{j}\right)+\frac{\partial}{\partial \theta}\left(v_{j} h_{j}\right)=0, \\
& p_{2}=p_{1}-h_{1}, \quad \varepsilon h_{1}+h_{2}=1+\varepsilon .
\end{aligned}
$$

where

$$
\varepsilon=\frac{\bar{h}_{1}}{\bar{h}_{2}}
$$

is the depth ratio. We shall also impose the usual smoothness conditions and the center of the vortex,

$$
\begin{array}{ll}
\frac{\partial u_{j}}{\partial \theta}-v_{j} \rightarrow 0, & \frac{\partial v_{j}}{\partial \theta}+u_{j} \rightarrow 0, \\
\frac{\partial p_{j}}{\partial \theta} \rightarrow 0 \quad \text { as } \quad r \rightarrow 0, & \frac{\partial h_{j}}{\partial \theta} \rightarrow 0,
\end{array}
$$

which guarantee that Eqs. (1)-(3) do not have a singularity at $r=0$.

Eqs. (1)-(4) admit an exact solution describing a steady axisymmetric vortex,

$$
u_{j}=0, \quad v_{j}=V_{j}(r), \quad p_{j}=P_{j}(r), \quad h_{j}=H_{j}(r),
$$

where the functions $V_{j}, P_{j}$, and $H_{j}$ satisfy the so-called cyclostrophic relations,

$$
\frac{\mathrm{d} P_{j}}{\mathrm{~d} r}=V_{j}+\frac{1}{r} V_{j}^{2}, \quad P_{2}=P_{1}-H_{1}, \quad \varepsilon H_{1}+H_{2}=1+\varepsilon .
$$

We shall assume that $V_{j}(r)$ are smooth functions, decaying as $r \rightarrow \infty$ and vanishing at $r=0$ (the latter condition guarantees that the vortex is smooth at its centre). Note also that the scaling of $h_{1}$ implies that

$$
H_{1} \rightarrow 1 \quad \text { as } \quad r \rightarrow \infty
$$

In order to examine the stability of vortex (6), seek a solution in the form

$$
\begin{aligned}
& u_{j}(r, \theta, t)=u_{j}^{\prime}(r, \theta, t), \quad v_{j}(r, \theta, t)=V_{j}(r)+v_{j}^{\prime}(r, \theta, t), \\
& p_{j}(r, \theta, t)=P_{j}(r)+p_{j}^{\prime}(r, \theta, t), \quad h_{j}(r, \theta, t)=H_{j}(r)+h_{j}^{\prime}(r, \theta, t),
\end{aligned}
$$

where the primed variables describe a small disturbance. Substituting (7)-(8) into (1)-(4), we obtain (primes omitted)

$$
\begin{aligned}
& \frac{\partial u_{j}}{\partial t}+\frac{1}{r} V_{j}\left(\frac{\partial u_{j}}{\partial \theta}-v_{j}\right)-\frac{1}{r} v_{j} V_{j}+\frac{\partial p_{j}}{\partial r}=v_{j}, \\
& \frac{\partial v_{j}}{\partial t}+u_{j} \frac{\partial V_{j}}{\partial r}+\frac{1}{r} V_{j}\left(\frac{\partial v_{j}}{\partial \theta}+u_{j}\right)+\frac{1}{r} \frac{\partial p_{j}}{\partial \theta}=-u_{j}, \\
& r \frac{\partial h_{j}}{\partial t}+\frac{\partial}{\partial r}\left(r u_{j} H_{j}\right)+\frac{\partial}{\partial \theta}\left(V_{j} h_{j}+v_{j} H_{j}\right)=0,
\end{aligned}
$$




$$
p_{2}=p_{1}-h_{1}, \quad \varepsilon h_{1}+h_{2}=0 .
$$

We shall consider eigenmodes, i.e. disturbances with harmonic dependence on $t$ and $\theta$,

$$
\begin{array}{ll}
u_{j}(r, \theta, t)=\tilde{u}_{j}(r) \mathrm{e}^{\mathrm{i} k \theta-\mathrm{i} \omega t}, & v_{j}(r, \theta, t)=\tilde{v}_{j}(r) \mathrm{e}^{\mathrm{i} k \theta-\mathrm{i} \omega t}, \\
p_{j}(r, \theta, t)=\tilde{p}_{j}(r) \mathrm{e}^{\mathrm{i} k \theta-\mathrm{i} \omega t}, & h_{j}(r, \theta, t)=\tilde{h}_{j}(r) \mathrm{e}^{\mathrm{i} k \theta-\mathrm{i} \omega t},
\end{array}
$$

where $\omega$ and $k$ are the frequency and azimuthal wavenumber. Substitution of (13) and (14) into (9)-(12) yields (tildes omitted)

$$
\begin{aligned}
& \mathrm{i}\left(\frac{k}{r} V_{j}-\omega\right) u_{j}-\left(1+\frac{2}{r} V_{j}\right) v_{j}+\frac{\mathrm{d} p_{j}}{\mathrm{~d} r}=0, \\
& \mathrm{i}\left(\frac{k}{r} V_{j}-\omega\right) v_{j}+\left(1+\frac{1}{r} V_{j}+\frac{\mathrm{d} V_{j}}{\mathrm{~d} r}\right) u_{j}+\frac{\mathrm{i} k}{r} p_{j}=0, \\
& \mathrm{i}\left(\frac{k}{r} V_{j}-\omega\right) h_{j}+\frac{1}{r} \frac{\mathrm{d}}{\mathrm{d} r}\left(r u_{j} H_{j}\right)+\frac{\mathrm{i} k}{r} v_{j} H_{j}=0, \\
& p_{2}=p_{1}-h_{1}, \quad \varepsilon h_{1}+h_{2}=0 .
\end{aligned}
$$

These boundary conditions for disturbances at the center of the vortex follow from (5),

$$
\mathrm{i} k u_{j}-v_{j} \rightarrow 0, \quad \mathrm{i} k v_{j}+u_{j} \rightarrow 0, \quad h_{j} \rightarrow 0, \quad p_{j} \rightarrow 0 \quad \text { as } \quad r \rightarrow 0 .
$$

We shall also impose the boundedness conditions at infinity,

$$
u_{j}, v_{j}, h_{j}, p_{j} \rightarrow 0 \quad \text { as } \quad r \rightarrow \infty .
$$

(15)-(20) form an eigenvalue problem, where $\omega$ is the eigenvalue. If $\operatorname{Im} \omega>0$, the vortex is unstable.

In some cases, it is convenient to eliminate $u_{j}$ and $v_{j}$. To do so, these variables should be expressed from (15) and (16) and substituted into (17), which yields

$$
\frac{\mathrm{d}}{\mathrm{d} r}\left(r F_{j} \frac{\mathrm{d} p_{j}}{\mathrm{~d} r}\right)-\left\{\frac{k^{2}}{r} F_{j}+\frac{\frac{\mathrm{d}}{\mathrm{d} r}\left[\frac{k}{r}\left(r+2 V_{j}\right) F_{j}\right]}{\omega-\frac{k}{r} V_{j}}\right\} p_{j}-r h_{j}=0,
$$

where

$$
F_{j}=\frac{H_{j}}{\left(1+\frac{1}{r} V_{j}+\frac{\mathrm{d} V_{j}}{\mathrm{~d} r}\right)\left(1+\frac{2}{r} V_{j}\right)-\left(\frac{k}{r} V_{j}-\omega\right)^{2}} .
$$

\section{Classification of the eigenmodes}

Consider a compensated vortex, i.e. put

$$
V_{2}=0 \text {. }
$$


To examine its stability, it is convenient to use Eqs. (15)-(17) $(j=1)$ for the upper layer and Eq. $(21)(j=2)$ for the lower layer, with the appropriate boundary conditions resulting from (19) and (20). Replacing $h_{1}$ with $p_{1}-p_{2}$ [see (19)], we obtain

$$
\begin{aligned}
& \mathrm{i}\left(\frac{k}{r} V_{1}-\omega\right) u_{1}-\left(1+\frac{2}{r} V_{1}\right) v_{1}+\frac{\mathrm{d} p_{1}}{\mathrm{~d} r}=0, \\
& \mathrm{i}\left(\frac{k}{r} V_{1}-\omega\right) v_{1}+\left(1+\frac{1}{r} V_{1}+\frac{\mathrm{d} V_{1}}{\mathrm{~d} r}\right) u_{1}+\frac{\mathrm{i} k}{r} p_{1}=0, \\
& \mathrm{i}\left(\frac{k}{r} V_{1}-\omega\right)\left(p_{1}-p_{2}\right)+\frac{1}{r} \frac{\mathrm{d}}{\mathrm{d} r}\left(r u_{1} H_{1}\right)+\frac{\mathrm{i} k}{r} v_{1} H_{1}=0, \\
& \mathrm{i} k u_{1}-v_{1} \rightarrow 0, \quad p_{1} \rightarrow 0 \quad \text { as } \quad r v_{1}+u_{1} \rightarrow 0, \quad r \rightarrow 0, \\
& u_{1}, v_{1}, p_{1} \rightarrow 0 \quad \text { as } \quad r \rightarrow \infty, \\
& \left.\quad \begin{array}{l}
\mathrm{d} \\
\mathrm{d} r
\end{array} r\left(1-\varepsilon H_{1}\right) \frac{\mathrm{d} p_{2}}{\mathrm{~d} r}\right]-\left[\frac{\omega k^{2}}{r}\left(1-\varepsilon H_{1}\right)-\varepsilon k \frac{\mathrm{d} H_{1}}{\mathrm{~d} r}\right] p_{2} \\
& \quad+\varepsilon \omega\left(1-\omega^{2}\right) r\left(p_{1}-p_{2}\right)=0 . \\
& p_{2} \rightarrow 0 \quad \text { as } \quad r \rightarrow 0, \infty .
\end{aligned}
$$

Next, substitution of (23) into the cyclostrophic relations (6) yields

$$
\frac{\mathrm{d} H_{1}}{\mathrm{~d} r}=V_{1}+\frac{1}{r} V_{1}^{2} .
$$

Everywhere in this paper, we assume that the upper layer is much thinner than the lower layer, i.e. $\varepsilon \ll 1$. Then, seek a solution in the form

$$
\begin{array}{ll}
u_{1}=u_{1}^{(0)}+\varepsilon u_{1}^{(1)}+\cdots & v_{1}=v_{1}^{(0)}+\varepsilon v_{1}^{(1)}+\cdots \quad p_{1}=p_{1}^{(0)}+\varepsilon p_{1}^{(1)}+\cdots \\
p_{2}=p_{2}^{(0)}+\varepsilon p_{2}^{(1)}+\cdots & \omega=\omega^{(0)}+\varepsilon \omega^{(1)}+\cdots
\end{array}
$$

First of all, consider the zeroth order of the lower layer problem (29) and (30),

$$
\begin{aligned}
& \omega^{(0)}\left[\frac{\mathrm{d}}{\mathrm{d} r}\left(r \frac{\mathrm{d} p_{2}^{(0)}}{\mathrm{d} r}\right)-\frac{k^{2}}{r} p_{2}^{(0)}\right]=0 . \\
& p_{2}^{(0)} \rightarrow 0 \quad \text { as } \quad r \rightarrow 0, \infty .
\end{aligned}
$$

Clearly, if $\omega^{(0)}, p_{2}^{(0)} \neq 0$, problem (32) and (33) has no solution. ${ }^{1}$ Hence, either $p_{2}^{(0)}$, or $\omega^{(0)}$, or both, have to be zero, depending on which we shall distinguish three types of modes:

\footnotetext{
${ }^{1}$ If $\omega^{(0)} \neq 0$, the solution of $(32)$ is $p_{2}^{(0)}=$ const $_{1} r^{k}+$ const $_{2} r^{-k}$, which never satisfies both boundary conditions (33).
} 
1. If

$$
p_{2}^{(0)}=0, \quad \omega^{(0)} \neq 0
$$

the upper layer problem (24)-(28), to leading order, decouples from the lower layer and describes the usual equivalent barotropic motion. Its solutions will be referred to as upper layer dominated (ULD) modes.

2. If

$$
p_{2}^{(0)} \neq 0, \quad \omega^{(0)}=0
$$

the lower layer problem (29)-(30), to leading order, decouples from the upper layer:

$$
\begin{aligned}
& \omega^{(1)} \frac{\mathrm{d}}{\mathrm{d} r}\left(r \frac{\mathrm{d} p_{2}^{(0)}}{\mathrm{d} r}\right)-\left(\frac{\omega^{(1)} k^{2}}{r}-k \frac{\mathrm{d} H_{1}}{\mathrm{~d} r}\right) p_{2}^{(0)}=0, \\
& p_{2}^{(0)} \rightarrow 0 \quad \text { as } \quad r \rightarrow 0, \infty .
\end{aligned}
$$

and fully determines $\omega^{(1)}$.

Eqs. (34)-(35) describe oscillations in a layer with non-even upper boundary, and its solutions will be referred to as lower layer dominated (LLD) modes. They exist due to the curvature of the interface and, to leading order, are not sensitive to the flow in the upper layer. The leading-order upper layer equations in turn, describe a disturbance forced by the pressure variations in the lower layer.

3. If

$$
p_{2}^{(0)}=0, \quad \omega^{(0)}=0
$$

the eigenvalue $\omega$, to leading order, drops out from the upper layer problem,

$$
\begin{aligned}
& \frac{\mathrm{i} k}{r} V_{1} u_{1}^{(0)}-\left(1+\frac{2}{r} V_{1}\right) v_{1}^{(0)}+\frac{\mathrm{d} p_{1}^{(0)}}{\mathrm{d} r}=0, \\
& \frac{\mathrm{i} k}{r} V_{1} v_{1}^{(0)}+\left(1+\frac{1}{r} V_{1}+\frac{\mathrm{d} V_{1}}{\mathrm{~d} r}\right) u_{1}^{(0)}+\frac{\mathrm{i} k}{r} p_{1}^{(0)}=0, \\
& \frac{\mathrm{i} k}{r} V_{1} p_{1}^{(0)}+\frac{1}{r} \frac{\mathrm{d}}{\mathrm{d} r}\left(r u_{1}^{(0)} H_{1}\right)+\frac{\mathrm{i} k}{r} v_{1}^{(0)} H_{1}=0 . \\
& \mathrm{i} k u_{1}^{(0)}-v_{1}^{(0)} \rightarrow 0, \quad \mathrm{i} k v_{1}^{(0)}+u_{1}^{(0)} \rightarrow 0, \quad h_{1}^{(0)} \rightarrow 0, \\
& p_{1}^{(0)} \rightarrow 0 \quad \text { as } \quad r \rightarrow 0, \quad \quad \text { as } \quad r \rightarrow \infty . \\
& u_{1}^{(0)}, v_{1}^{(0)}, p_{1}^{(0)} \rightarrow 0 \quad
\end{aligned}
$$


$\omega^{(1)}$ is determined by the next-order equations:

$$
\begin{aligned}
& -\mathrm{i} \omega^{(1)} u_{1}^{(0)}+\frac{\mathrm{i} k}{r} V_{1} u_{1}^{(1)}-\left(1+\frac{2}{r} V_{1}\right) v_{1}^{(1)}+\frac{\mathrm{d} p_{1}^{(1)}}{\mathrm{d} r}=0, \\
& -\mathrm{i} \omega^{(1)} v_{1}^{(0)}+\frac{\mathrm{i} k}{r} V_{1} v_{1}^{(1)}+\left(1+\frac{1}{r} V_{1}+\frac{\mathrm{d} V_{1}}{\mathrm{~d} r}\right) u_{1}^{(1)}+\frac{\mathrm{i} k}{r} p_{1}^{(1)}=0, \\
& -\mathrm{i} \omega^{(1)} p_{1}^{(0)}+\frac{\mathrm{i} k}{r} V_{1}\left(p_{1}^{(1)}-p_{2}^{(1)}\right)+\frac{1}{r} \frac{\mathrm{d}}{\mathrm{d} r}\left(r u_{1}^{(1)} H_{1}\right)+\frac{\mathrm{i} k}{r} v_{1}^{(1)} H_{1}=0, \\
& \omega^{(1)} \frac{\mathrm{d}}{\mathrm{d} r}\left(r \frac{\mathrm{d} p_{2}^{(1)}}{\mathrm{d} r}\right)-\left(\frac{\omega^{(1)} k^{2}}{r}-k \frac{\mathrm{d} H_{1}}{\mathrm{~d} r}\right) p_{2}^{(1)}+\omega^{(1)} r p_{1}^{(0)}=0 .
\end{aligned}
$$

Importantly, (41)-(44) include characteristics of both layers-hence, the corresponding solutions will be referred to as mixed $(M)$ modes.

Observe that the last two types of modes are slow $\left(\omega \approx \varepsilon \omega^{(1)} \ll 1\right)$ and are responsible for baroclinic instability-whereas fast ULD-modes $\left(\omega \approx \omega^{(0)} \sim 1\right)$ are responsible for equivalent-barotropic instability. Thus, a barotropically unstable ring would disintegrate on the timescale of $f_{0}^{-1}$, i.e. within days. It has also been shown (Benilov, 2003) that, at least for quasigeostrophic rings, ULD-modes exist only for vortices with a radius less than half of the deformation radius. Given that the latter is, typically, 10-50 km, ULD-modes do not play an important role for mesoscale oceanic rings, and we shall concentrate on the other two types.

Observe also that the above types of eigenmodes are similar to their QG analogues (see Benilov, 2003). This can be partly explained by the fact that, for the two slow types of modes, the lower layer flow is quasigeostrophic (indeed, since the upper layer is thin, the displacement of the interface is much smaller than the lower layer's depth). It should be emphasized, however, that the upper layer is ageostrophic in all three cases.

Finally, note that our classification does not include the eigenemodes associated with radiation of internal waves - the (dimensional) frequency of these modes is much greater than $f_{0}$, which makes them inconsistent with out scaling. These modes have been studied by Ford (1994), Plougonven and Zeitlen (2002), and Schecter and Montgomery (2004).

\section{Mixed modes}

In this section, we shall examine the stability of a compensated vortex with respect to M-modes. LLD-modes will be examined in Section 5. 


\subsection{Leading-order results}

The zeroth-order problem (36)-(40) appears to have a solution only for the first azimuthal wavenumber, ${ }^{2}$

$$
k=1 \text {, }
$$

in which case, one can verify by inspection that

$$
u_{1}^{(0)}=-\frac{\mathrm{i}}{r} V_{1}, \quad v_{1}^{(0)}=\frac{\mathrm{d} V_{1}}{\mathrm{~d} r}, \quad p_{1}^{(0)}=\frac{\mathrm{d} H_{1}}{\mathrm{~d} r} .
$$

Strictly speaking, in order to justify linearization, solution (45) should be multiplied by a small constant-however, given the linearity of the problem, this constant can be omitted.

Substituting (45) into the first-order upper layer Eqs. (41)-(43), we obtain

$$
\begin{aligned}
& \frac{\mathrm{i}}{r} V_{1} u_{1}^{(1)}-\left(1+\frac{2}{r} V_{1}\right) v_{1}^{(1)}+\frac{\mathrm{d} p_{1}^{(1)}}{\mathrm{d} r}=\frac{\omega^{(1)}}{r} V_{1}, \\
& \frac{\mathrm{i}}{r} V_{1} v_{1}^{(1)}+\left(1+\frac{1}{r} V_{1}+\frac{\mathrm{d} V_{1}}{\mathrm{~d} r}\right) u_{1}^{(1)}+\frac{\mathrm{i}}{r} p_{1}^{(1)}=\mathrm{i} \omega^{(1)} \frac{\mathrm{d} V_{1}}{\mathrm{~d} r}, \\
& \frac{\mathrm{i}}{r} V_{1} p_{1}^{(1)}+\frac{1}{r} \frac{\mathrm{d}}{\mathrm{d} r}\left(r u_{1}^{(1)} H_{1}\right)+\frac{\mathrm{i}}{r} v_{1}^{(1)} H_{1}=\mathrm{i} \omega^{(1)} \frac{\mathrm{d} H_{1}}{\mathrm{~d} r}+\frac{\mathrm{i}}{r} V_{1} p_{2}^{(1)},
\end{aligned}
$$

and the corresponding boundary conditions follow from the general conditions (27)-(28),

$$
\begin{array}{ll}
\mathrm{i} v_{1}^{(1)}+u_{1}^{(1)} \rightarrow 0, & p_{1}^{(1)} \rightarrow 0 \quad \text { as } \quad r \rightarrow 0, \\
u_{1}^{(1)}, v_{1}^{(1)}, p_{1}^{(1)} \rightarrow 0 \quad \text { as } \quad r \rightarrow \infty . &
\end{array}
$$

Eqs. (46)-(50) form a non-homogeneous boundary-value problem for $u_{1}^{(1)}, v_{1}^{(1)}, p_{1}^{(1)}$ — which has a solution only subject to a certain orthogonality condition. To derive this condition, consider

$$
\int_{0}^{\infty}\left[(46) \times \mathrm{i} r H_{1}+(47) \times r H_{1}+(48) \times r\left(r+V_{1}\right)\right] \mathrm{d} r .
$$

Straightforward calculations, involving integration by parts and use of the boundary conditions, yield

$$
\omega^{(1)} \int_{0}^{\infty} r^{2} \frac{\mathrm{d} H_{1}}{\mathrm{~d} r} \mathrm{~d} r+\int_{0}^{\infty} r \frac{\mathrm{d} H_{1}}{\mathrm{~d} r} p_{2}^{(1)} \mathrm{d} r=0 .
$$

\footnotetext{
${ }^{2}$ Unfortunately, we have been unable to rigorously prove that no solution exists for $k \geq 2$. Instead, an extensive numerical study has been carried out: for various vortex profiles, (36)-(40) were solved as an eigenvalue problem, with $k$ being the eigenvalue. In all cases, the only solution found was $k=1$. It is also worth mentioning that, for the QG analogue of (36)-(40), the non-existence of solutions for $k \geq 2$ can be proved rigorously (Benilov, 2003).
} 
Finally, substitute $k=1$ and (45) into the first-order lower layer Eq. (44),

$$
\omega^{(1)} \frac{\mathrm{d}}{\mathrm{d} r}\left(r \frac{\mathrm{d} p_{2}^{(1)}}{\mathrm{d} r}\right)-\left(\frac{\omega^{(1)}}{r}-\frac{\mathrm{d} H_{1}}{\mathrm{~d} r}\right) p_{2}^{(1)}+\omega^{(1)} r \frac{\mathrm{d} H_{1}}{\mathrm{~d} r}=0,
$$

which is to be solved with

$$
p_{2}^{(1)} \rightarrow 0 \quad \text { as } \quad r \rightarrow 0, \infty
$$

(51)-(53) form an eigenvalue problem for $p_{2}^{(1)}$ and $\omega^{(1)}$, and it is identical to its QG analogue (see Benilov, 2003)!

We comment that the coincidence of Eq. (52) with its QG counterpart is not all that surprising, as the flow in the lower layer is indeed quasigeostrophic (see the comment in the end of Section 3). But the upper layer is ageostrophic-therefore, the coincidence of the (upper layer) Eq. (51) with its QG counterpart does come as a surprise.

The similarity between the present problem and its QG analogue enables us to use the properties of (51)-(53) established by Benilov (2003):

1. problem (51)-(53) admits infinitely many real eigenvalues $\omega^{(1)}$ converging to zero;

2. if $\frac{\mathrm{d} H_{1}(r)}{\mathrm{d} r}$ is sign definite (which is always the case oceanographically), all eigenvalues have the same sign as $\frac{\mathrm{d} H_{1}(r)}{\mathrm{d} r}$.

Thus, Property 1 guarantees that all M-modes are, to leading order, neutrally stable.

\subsection{Higher order results: the effect of critical levels}

As follows from the cyclostrophic relation (31), the angular velocity $\frac{1}{r} V_{1}$ has the same sign as $\frac{\mathrm{d} H_{1}}{\mathrm{~d} r}$-hence, due to Property 2 above, it has the same sign as the frequency $\omega$. Observe also that $\frac{1}{r} V_{1}$ varies from zero (at infinity) to an order-one value (in the core of the vortex), whereas $\omega \approx \varepsilon \omega^{(1)}$ is small. Then, there exists a critical level (radius), i.e. a point $r=r_{\mathrm{c}}$ such that

$$
\frac{k}{r_{\mathrm{c}}} V_{1}\left(r_{\mathrm{c}}\right)=\operatorname{Re} \omega
$$

Most importantly, the smallness of $\omega$ implies that the critical level is located where $\frac{1}{r} V_{1}$ is also small, i.e. at the periphery of the vortex.

Note also that, in the leading-order Eqs. (36)-(38), the terms involving $\omega$ were omitted, and those involving $\frac{1}{r} V_{1}$, retained-which is clearly inconsistent near the critical level. We conclude that our expansion describes the critical level incorrectly.

Still, the fact that the critical level has not been 'captured' by the leading-order expansion indicates that its effect is weak - after all, the critical level is located at the periphery of the vortex, where the disturbance rapidly decays. We can safely assume that its contribution to the eigenvalue is small — but no matter how small, it can be imaginary and, hence, cause 
instability. Mathematically, this means that one of the higher order corrections to $\omega$ can be complex.

To take into account the effect of critical levels, we shall use an asymptotic approach used by Schecter et al. (2001), Schecter and Montgomery (2003), and Benilov (2003) for similar vortex problems. It is applicable to the present problem as well-but, unfortunately, is associated with extremely cumbersome algebra. Accordingly, non-mathematically minded readers are advised to jump to the next subsection, where the asymptotic results are summarized. First of all, use (18) to eliminate $h_{j}$ from Eq. (21), which then yields

$$
\begin{aligned}
& \frac{\mathrm{d}}{\mathrm{d} r}\left(r F_{1} \frac{\mathrm{d} p_{1}}{\mathrm{~d} r}\right)-\left\{\frac{k^{2}}{r} F_{1}+\frac{\frac{\mathrm{d}}{\mathrm{d} r}\left[\frac{k}{r}\left(r+2 V_{1}\right) F_{1}\right]}{\omega-\frac{k}{r} V_{1}}\right\} p_{1}+r\left(p_{2}-p_{1}\right)=0, \\
& \frac{\mathrm{d}}{\mathrm{d} r}\left(r F_{2} \frac{\mathrm{d} p_{2}}{\mathrm{~d} r}\right)-\left\{\frac{k^{2}}{r} F_{2}+\frac{\frac{\mathrm{d}}{\mathrm{d} r}\left[\frac{k}{r}\left(r+2 V_{2}\right) F_{2}\right]}{\omega-\frac{k}{r} V_{2}}\right\} p_{2}+\varepsilon r\left(p_{1}-p_{2}\right)=0,
\end{aligned}
$$

where $F_{1,2}$ are defined by (22). Next, consider

$$
\operatorname{Im} \int_{0}^{\infty}\left[\varepsilon(54)_{j=1} p_{1}^{*}+(55)_{j=2} p_{2}^{*}\right] \mathrm{d} r
$$

where the asterisk denotes complex conjugate. Integrating by parts, using boundary conditions (19)-(20), and putting $V_{2}=0$, we obtain

$$
\begin{aligned}
& \int_{0}^{\infty} r\left[F_{\mathrm{I}}\left(\left|\frac{\mathrm{d} p_{1}}{\mathrm{~d} r}\right|^{2}+\frac{k^{2}}{r^{2}}\left|p_{1}\right|^{2}\right)-2 H_{1} \frac{\omega_{\mathrm{R}} \omega_{\mathrm{I}}}{\left|1-\omega^{2}\right|^{2}}\left(\left|\frac{\mathrm{d} p_{2}}{\mathrm{~d} r}\right|^{2}+\frac{k^{2}}{r^{2}}\left|p_{2}\right|^{2}\right)\right] \mathrm{d} r \\
& -\int_{0}^{\infty} \frac{\omega_{\mathrm{I}} \frac{\mathrm{d}}{\mathrm{d} r}\left[\frac{k}{r}\left(r+2 V_{1}\right) F_{\mathrm{R}}\right]+\left(\frac{k}{r} V_{1}-\omega_{\mathrm{R}}\right) \frac{\mathrm{d}}{\mathrm{d} r}\left[\frac{k}{r}\left(r+2 V_{1}\right) F_{\mathrm{I}}\right]}{\left(\omega_{\mathrm{R}}-\frac{k}{r} V_{1}\right)^{2}+\omega_{\mathrm{I}}^{2}} \\
& \times\left|p_{1}\right|^{2} \mathrm{~d} r+k \omega_{\mathrm{I}} \frac{1-3 \omega_{\mathrm{R}}^{2}+\omega_{\mathrm{I}}^{2}}{\left|\omega\left(1-\omega^{2}\right)\right|^{2}} \int_{0}^{\infty} \frac{\mathrm{d} H_{1}}{\mathrm{~d} r}\left|p_{2}\right|^{2} \mathrm{~d} r=0,
\end{aligned}
$$

where

$$
F_{\mathrm{R}}=\operatorname{Re} F_{1}, \quad F_{\mathrm{I}}=\operatorname{Im} F_{1}, \quad \omega_{\mathrm{R}}=\operatorname{Re} \omega, \quad \omega_{\mathrm{I}}=\operatorname{Im} \omega .
$$

In what follows, (56) will be simplified and, eventually, transformed into an equation for $\omega_{I}$.

Since we deal with M-modes, we can put

$$
k=1, \quad \omega_{\mathrm{R}} \approx \varepsilon \omega^{(1)},
$$


where $\omega^{(1)}$ is determined by problem (51)-(53). Then, recall that, to leading order, $\omega$ turned out to be real, which implies

$$
\left|\omega_{\mathrm{I}}\right| \ll\left|\omega_{\mathrm{R}}\right| .
$$

Now, we can reduce $(22)(j=1)$ to

$$
F_{\mathrm{R}} \approx \frac{H_{1}}{B}, \quad F_{\mathrm{I}} \approx-\frac{2 V_{1} H_{1}}{r B^{2}} \omega_{\mathrm{I}},
$$

where

$$
B=1+\frac{3}{r} V_{1}+\frac{1}{r^{2}} V_{1}^{2}+\frac{\mathrm{d} V_{1}}{\mathrm{~d} r}+\frac{2}{r} V_{1} \frac{\mathrm{d} V_{1}}{\mathrm{~d} r} .
$$

To further simplify Eq. (56), we shall use the leading-order approximations of the eigenfunctions,

$$
p_{1} \approx p_{1}^{(0)}=\frac{\mathrm{d} H}{\mathrm{~d} r}, \quad p_{2} \approx \varepsilon p_{2}^{(1)},
$$

where the former is given by (45), and the latter is determined by (51)-(53). Note that equalities (60) are not valid near the critical level-recalling that that is located at the periphery of the vortex, we subdivide the region of integration in (56) into core and periphery,

$$
\frac{k}{r} V_{1} \gg \omega_{\mathrm{R}} \quad \text { if } \quad r<r_{\mathrm{b}}
$$

and

$$
\frac{k}{r} V_{1} \lesssim \omega_{\mathrm{R}} \quad \text { if } \quad r>r_{\mathrm{b}}
$$

where $r_{\mathrm{b}}$ is an approximate boundary separating the two regions. Observe, however, that this needs to be done only in the second term of (56), which involves

$$
\frac{1}{\left(\omega_{\mathrm{R}}-(k / r) V_{1}\right)^{2}+\omega_{\mathrm{I}}^{2}} .
$$

Indeed, near the critical level, this factor becomes large and, hence, increases the error resulting from the 'incorrect' expression for $p_{1}$-whereas the errors introduced by the other two terms of (56) are much smaller.

Now, take into account (57), separate (where necessary) the core from periphery, and use (60) in the former-after which (56) becomes

$$
\varepsilon \omega_{\mathrm{I}} I_{\text {core }}+I_{\text {peri }}=0,
$$

where

$$
I_{\text {core }} \approx-\int_{0}^{\infty} r \frac{2 V_{1} H_{1}}{r B^{2}}\left[\left(\frac{\mathrm{d} H_{1}}{\mathrm{~d} r^{2}}\right)^{2}+\frac{1}{r^{2}}\left(\frac{\mathrm{d} H_{1}}{\mathrm{~d} r}\right)^{2}\right] \mathrm{d} r
$$




$$
\begin{aligned}
& -\int_{0}^{r_{\mathrm{b}}} \frac{\frac{\mathrm{d}}{\mathrm{d} r}\left[\frac{1}{r}\left(r+2 V_{1}\right) \frac{H_{1}}{B}\right]-\frac{1}{r} V_{1} \frac{\mathrm{d}}{\mathrm{d} r}\left[\frac{1}{r}\left(r+2 V_{1}\right) \frac{2 V_{1} H_{1}}{r B^{2}}\right]}{\frac{1}{r^{2}} V_{1}^{2}}\left(\frac{\mathrm{d} H_{1}}{\mathrm{~d} r}\right)^{2} \mathrm{~d} r \\
& +\frac{1}{\omega^{(1) 2}} \int_{0}^{\infty} \frac{\mathrm{d} H_{1}}{\mathrm{~d} r} p_{2}^{(1) 2} \mathrm{~d} r, \\
& I_{\text {peri }} \approx-\int_{r_{b}}^{\infty} \frac{\omega_{I} \frac{\mathrm{d}}{\mathrm{d} r}\left[\frac{1}{r}\left(r+2 V_{1}\right) \frac{H_{1}}{B}\right]-\left(\frac{1}{r} V_{1}-\omega_{R}\right) \frac{\mathrm{d}}{\mathrm{d} r}\left[\frac{1}{r}\left(r+2 V_{1}\right) \frac{2 V_{1} H_{1}}{r B^{2}}\right]}{\left(\omega_{R}-\frac{1}{r} V_{1}\right)+\omega_{I}^{2}}\left|p_{1}\right|^{2} \mathrm{~d} r .
\end{aligned}
$$

Now, observe that integration in the second term of $I_{\text {core }}$ can be extended to infinity (which is allowable since the integrand decays rapidly as $r \rightarrow \infty$ ). Then, the first two terms can be grouped together and rearranged using the cyclostrophic relation (31) and integration by parts,

$$
\begin{aligned}
I_{\text {core }}= & -\int_{0}^{\infty} \frac{2 V_{1} H_{1}}{r B^{2}}\left\{r\left(\frac{\mathrm{d} H_{1}}{\mathrm{~d} r^{2}}\right)^{2}+\frac{1}{r}\left(\frac{\mathrm{d} H_{1}}{\mathrm{~d} r}\right)^{2}+\left(1+\frac{2}{r} V_{1}\right) \frac{\mathrm{d}}{\mathrm{d} r}\left[\frac{1}{r} V_{1}\left(r+V_{1}\right)^{2}\right]\right\} \mathrm{d} r \\
& +\int_{0}^{\infty}\left[\left(1+\frac{2}{r} V_{1}\right) \frac{H_{1}}{B}-1\right] \frac{\mathrm{d}}{\mathrm{d} r}\left[\left(r+V_{1}\right)^{2}\right] \mathrm{d} r+\frac{1}{\omega^{(1) 2}} \int_{0}^{\infty} \frac{\mathrm{d} H_{1}}{\mathrm{~d} r} p_{2}^{(1) 2} \mathrm{~d} r
\end{aligned}
$$

(it is implied here that $H_{1} \rightarrow 1$ as $r \rightarrow \infty$ ). Then, taking into account the identity

$$
\begin{aligned}
& r\left(\frac{\mathrm{d} H_{1}}{\mathrm{~d} r^{2}}\right)^{2}+\frac{1}{r}\left(\frac{\mathrm{d} H_{1}}{\mathrm{~d} r}\right)^{2}+\left(1+\frac{2}{r} V_{1}\right) \frac{\mathrm{d}}{\mathrm{d} r}\left[\left(r+V_{1}\right)\left(V_{1}+\frac{1}{r} V_{1}^{2}\right)\right] \\
& \quad=B \frac{\mathrm{d}}{\mathrm{d} r}\left(r V_{1}+V_{1}^{2}\right)
\end{aligned}
$$

(which can be verified using the cyclostrophic relation and definition (59) of $B$ ), we obtain

$$
I_{\text {core }}=-\int_{0}^{\infty} r^{2} \frac{\mathrm{d} H_{1}}{\mathrm{~d} r} \mathrm{~d} r+\int_{0}^{\infty} \frac{\mathrm{d} H_{1}}{\mathrm{~d} r}\left(\frac{p_{2}^{(1)}}{\omega^{(1)}}\right)^{2} \mathrm{~d} r .
$$

Finally, rewrite equality (51) as follows:

$$
2 \int_{0}^{\infty} r^{2} \frac{\mathrm{d} H_{1}}{\mathrm{~d} r} \mathrm{~d} r+\int_{0}^{\infty} 2 r \frac{\mathrm{d} H_{1}}{\mathrm{~d} r} \frac{p_{2}^{(1)}}{\omega^{(1)}} \mathrm{d} r=0
$$

and use it to rewrite (62) in the form

$$
I_{\text {core }}=\int_{0}^{\infty} \frac{\mathrm{d} H_{1}}{\mathrm{~d} r}\left(r+\frac{p_{2}^{(1)}}{\omega^{(1)}}\right)^{2} \mathrm{~d} r
$$


Next, to simplify $I_{\text {peri }}$, observe that the main contribution to this term comes from the region near the critical level, where the denominator of the integrand is small. Hence, we can:

1. expand the integrand about $r=r_{\mathrm{c}}$,

2. and extend integration to infinity (since the integrand decays rapidly with distance from $r=r_{\mathrm{c}}$, this will cause only a small error).

Then, we obtain

$$
\begin{gathered}
I_{\text {peri }} \approx-\left\{\left|p_{1}\right|^{2} \frac{\mathrm{d}}{\mathrm{d} r}\left[\frac{1}{r}\left(r+2 V_{1}\right) \frac{H_{1}}{B}\right]\right\}_{r=r_{\mathrm{c}}} \\
\times \int_{-\infty}^{\infty} \frac{\omega_{\mathrm{I}} \mathrm{d} r}{\left\{\left[\frac{\mathrm{d}}{\mathrm{d} r}\left(\frac{1}{r} V_{1}\right)\right]_{r=r_{\mathrm{c}}}\left(r-r_{\mathrm{c}}\right)\right\}^{2}+\omega_{\mathrm{I}}^{2}} .
\end{gathered}
$$

The integral in (63) can be evaluated, and the rest of the expression can be simplified by taking into account that, in the periphery,

$$
\left|H_{1}-1\right| \ll 1
$$

Hence, the flow is weak, and the cyclostrophic relation reduces to the geostrophic one, $V_{1} \approx \frac{\mathrm{d} H_{1}}{\mathrm{~d} r}$. Eventually, we obtain

$$
I_{\text {peri }} \approx-\pi \operatorname{sign}\left(\omega_{I}\right)\left[\frac{V_{1}-\frac{1}{r} \frac{\mathrm{d}}{\mathrm{d} r}\left(r \frac{\mathrm{d} V_{1}}{\mathrm{~d} r}\right)+\frac{1}{r^{2}} V_{1}}{\left|\frac{\mathrm{d}}{\mathrm{d} r}\left(\frac{1}{r} V_{1}\right)\right|}\left|p_{1}\right|^{2}\right]_{r=r_{\mathrm{c}}} .
$$

Now, substituting (62) and (64) into (61), we obtain

$$
\left|\omega_{\mathrm{I}}\right| \int_{0}^{\infty} \frac{\mathrm{d} H_{1}}{\mathrm{~d} r}\left(r+\frac{p_{2}^{(1)}}{\omega^{(1)}}\right)^{2} \mathrm{~d} r=-\pi\left[\frac{Q_{1}^{\prime}}{\left|\frac{\mathrm{d}}{\mathrm{d} r}\left(\frac{1}{r} V_{1}\right)\right|}\left|p_{1}\right|^{2}\right]_{r=r_{\mathrm{c}}},
$$

where

$$
Q_{1}^{\prime}=\frac{1}{r} \frac{\mathrm{d}}{\mathrm{d} r}\left(r \frac{\mathrm{d} V_{1}}{\mathrm{~d} r}\right)-\frac{1}{r^{2}} V_{1}-V_{1}
$$

is the the upper layer PV gradient. $^{3}$

\footnotetext{
3 To be precise, we should call $Q_{1}^{\prime}$ quasigeostrophic PV gradient-but, in the periphery of the vortex, the exact and QG expressions for PV are approximately equal.
} 
Eq. (65) should be treated as an equation for $\omega_{I}$. As before, $\frac{\mathrm{d} H_{1}}{\mathrm{~d} r}$ is assumed to be a sign-definite function of $r$, in which case (65) has a solution if and only if $\frac{\mathrm{d} H_{1}}{\mathrm{~d} r}$ and $\left(Q_{1}^{\prime}\right)_{r=r_{\mathrm{c}}}$ are of opposite signs. If they indeed are, two solutions exist for $\omega_{\mathrm{I}}$, corresponding to two complex-conjugate eigenvalues, one of which is unstable. If, on the other hand, $\frac{\mathrm{d} H_{1}}{\mathrm{~d} r}$ and $\left(Q_{1}^{\prime}\right)_{r=r_{\mathrm{c}}}$ are of the same sign, no solutions exist for $\omega_{\mathrm{I}}$. This means that our boundaryvalue problem does not have eigenvalues (stable or unstable), which should be interpreted as stability.

Observe, however, that the right-hand side of (65) depends on the unknown quantities $r_{\mathrm{c}}$ and $\left(p_{1}\right)_{r=r_{\mathrm{c}}}$, which do not allow one to actually find $\omega_{\mathrm{I}} . r_{\mathrm{c}}$ can be determined approximately from the equation

$$
\left(\frac{k}{r} V_{1}\right)_{r=r_{\mathrm{c}}} \approx \varepsilon \omega^{(1)},
$$

whereas finding $\left(p_{1}\right)_{r=r_{\mathrm{c}}}$ is less straightforward, as its leading-order approximation (45) is inapplicable near the critical level.

However, if we use (65) as a stability criterion, the explicit expression for $\left(p_{1}\right)_{r=r_{\mathrm{c}}}$ will not be needed.

\subsection{Summary of the asymptotic results}

Given the profile $H_{1}(r)$ of a compensated vortex, its stability with respect to M-modes can be examined through the following steps:

1. solve eigenvalue problem (51)-(53) and find the leading-order frequency $\omega^{(1)}$,

2. substitute $\omega^{(1)}$ into (67) and determine the position of the critical level $r_{\mathrm{c}}$,

3. check the sign of the upper layer PV gradient at $r=r_{\mathrm{c}}$-if it is the same as that of $\frac{\mathrm{d} H_{1}}{\mathrm{~d} r}$, the vortex is stable with respect to M-modes (and vice versa).

Note that the above criterion implies that $\frac{\mathrm{d} H_{1}}{\mathrm{~d} r}$ does not change sign, i.e. $H_{1}(r)$ is a monotonic function. Observe also that, to ensure convergence of the integral on the lefthand side of (65), we should require

$$
\int_{0}^{\infty} \frac{\mathrm{d} H_{1}}{\mathrm{~d} r} r^{2} \mathrm{~d} r<\infty
$$

or, equivalently,

$$
\int_{0}^{\infty}\left(H_{1}-1\right) r \mathrm{~d} r<\infty .
$$

This condition is not very restrictive, as it simply guarantees that the net 'mass' of the vortex is finite.

Physical aspects of the above results will be discussed in Section 6 . 


\section{Lower layer dominated modes}

This case is similar to that of M-modes, and we shall not dwell on it in detail. We shall only mention that LLD-modes may exist only for $k \geq 2$ and, in this sense, complement M-modes (which may exist only for $k=1$ ). The LLD equivalent of the M-mode stability criterion (65) has the form

$$
k|\operatorname{Im} \omega| \int_{0}^{\infty} \frac{\mathrm{d} H_{1}}{\mathrm{~d} r}\left(\frac{p_{2}^{(0)}}{\omega^{(1)}}\right)^{2} \mathrm{~d} r=-\pi \varepsilon^{2}\left[\frac{Q_{1}^{\prime}}{\left|(\mathrm{d} / \mathrm{d} r)\left((1 / r) V_{1}\right)\right|}\left|p_{1}\right|^{2}\right]_{r=r_{\mathrm{c}}},
$$

where $p_{2}^{(0)}$ and $\omega^{(1)}$ satisfy the leading-order problem (34)-(35). Hence, the stability criterion for LLD-modes is exactly the same as that for M-modes: the vortex is stable if and only if the sign of the upper layer PV gradient $\left(Q_{1}^{\prime}\right)_{r=r_{\mathrm{c}}}$ coincides with that of $\frac{\mathrm{d} H_{1}}{\mathrm{~d} r}$.

\section{Discussion and concluding remarks}

We have derived the following stability criterion for a compensated vortex with a monotonic profile $H_{1}(r)$, in a two-layer ocean with thin upper layer:

If the sign of the upper layer PV gradient of the vortex at the critical level is the same as that of $\frac{\mathrm{d} H_{1}}{\mathrm{~d} r}$, the vortex is baroclinically stable and vice versa.

This criterion implies the knowledge of the position of the critical level—which is determined by problems (51)-(53), (34) and (35) (for mixed and lower layer dominated modes, respectively), and Eq. (67).

The most remarkable feature of the above criterion is that it coincides exactly with its QG analogue (Benilov, 2003). The coincidence can be partly explained by the fact that, since the upper layer is thin, the displacement of the interface is much smaller than the depth of the lower layer-hence, the flow there is indeed quasigeostrophic. It should be emphasized, however, that the flow in the upper layer is ageostrophic.

Note that the leading-order M and LLD problems, (51)-(53) and (34)-(35), are invariant with respect to simultaneous change

$$
\begin{aligned}
& \frac{\mathrm{d} H_{1}}{\mathrm{~d} r} \rightarrow \text { const } \times \frac{\mathrm{d} H_{1}}{\mathrm{~d} r}, \\
& \omega^{(1)} \rightarrow \text { const } \times \omega^{(1)} .
\end{aligned}
$$

Note also that, at the periphery of the vortex, velocity $V_{1}$ is weak and the (non-linear) cyclostrophic relation reduces to the (linear) quasigeostrophic one, while the exact expression for the PV gradient $Q_{1}^{\prime}$ reduces to the (linear) QG formula (66). As a result, change (69) entails

$$
\begin{aligned}
& V_{1} \rightarrow \text { const } \times V_{1} \quad \text { at the periphery of the vortex, } \\
& Q_{1}^{\prime} \rightarrow \text { const } \times Q_{1}^{\prime} \quad \text { at the periphery of the vortex. }
\end{aligned}
$$

Now, (70) and (71) indicate that the position of the critical level remains approximately the same, whereas (69) and (72) guarantee that the relative signs of $\left(Q_{1}^{\prime}\right)_{r=r_{\mathrm{c}}}$ and $\frac{\mathrm{d} H_{1}}{\mathrm{~d} r}$ remain 
the same. Hence, if a vortex with a certain profile $\frac{\mathrm{d} H_{1}(r)}{\mathrm{d} r}$ is baroclinically stable, then any profile of the form const $\times \frac{\mathrm{d} H_{1}(r)}{\mathrm{d} r}$ is stable [as long as it complies with the thin-upper layer requirement and corresponds to a positive $\left.H_{1}(r)\right] .{ }^{4}$ In other words, whether a vortex, in an ocean with thin upper layer, is stable or not, is determined by its shape, not amplitude (although the growth rate of an unstable vortex does depend on its amplitude).

Finally, observe that the similarity between quasigeostrophic and ageostrophic vortices applies only to baroclinic instability, as the above stability criterion does not include upper layer dominated (equivalent barotropic) disturbances. On the other hand, equivalent barotropic instability does not seem to be overly important for mesoscale oceanic rings, as it affects only small vortices (Benilov, 2003).

\section{References}

Benilov, E.S., 2003. Instability of quasigeostrophic vortices in a two-layer ocean with thin upper layer. J. Fluid Mech. 475, 303-331.

Benilov, E.S., 2004. Stability of vortices in a two-layer ocean with uniform potential vorticity in the lower layer. J. Fluid Mech. 502, 207-232.

Dewar, W.K., Killworth, P.D., 1995. On the stability of oceanic rings. J. Phys. Oceanogr. 25, 1467-1487.

Ford, R., 1994. The response of a rotating ellipse of uniform potential vorticity to gravity wave radiation. Phys. Fluids 6, 3664-3704.

Plougonven, R., Zeitlen, V., 2002. Internal gravity wave emission from a pancake vortex: an example of wavevortex interaction in strongly stratified flows. Phys. Fluids 14, 1259-1268.

Schecter, D.A., Montgomery, M.T., 2003. On the symmetrization rate of an intense geophysical vortex. Dyn. Atmos. Oceans 37, 55-88.

Schecter, D.A., Montgomery, M.T., 2004. Damping and pumping of a vortex Rossby wave in a monotonic cyclone: critical layer stirring versus inertia-buoyancy wave emission. Phys. Fluids 16, 1334-1348.

Schecter, D.A., Montgomery, M.T., Reasor, P.D., 2001. A theory for the vertical alignment of a quasigeostrophic vortex. J. Atmos. Sci. 59, 150-168.

\footnotetext{
${ }^{4}$ The same does not apply to the swirl velocity $V_{1}$, as, in the core of the vortex, it is related to $\frac{\mathrm{d} H_{1}}{\mathrm{~d} r}$ by the non-linear (cyclostrophic) relation (31).
} 